\title{
Sepsis 2019: What Surgeons Need to Know
}

\author{
Vanessa P. Ho, ${ }^{1}$ Haytham Kaafarani, ${ }^{2}$ Rishi Rattan, ${ }^{3}$ Nicholas Namias, ${ }^{3}$ \\ Heather Evans, ${ }^{4}$ and Tanya L. Zakrison ${ }^{5}$
}

\begin{abstract}
The definition of sepsis continues to be as dynamic as the management strategies used to treat this. Sepsis-3 has replaced the earlier systemic inflammatory response syndrome (SIRS)-based diagnoses with the rapid Sequential Organ Failure Assessment (SOFA) score assisting in predicting overall prognosis with regards to mortality. Surgeons have an important role in ensuring adequate source control while recognizing the threat of carbapenem-resistance in gram-negative organisms. Rapid diagnostic tests are being used increasingly for the early identification of multi-drug-resistant organisms (MDROs), with a key emphasis on the multidisciplinary alert of results. Novel, higher generation antibiotic agents have been developed for resistance in ESKCAPE (Enterococcus faecium, Staphylococcus aureus, Klebsiella pneumoniae, Acinetobacter baumannii, Pseudomonas aeruginosa, and Enterobacter species) organisms while surgeons have an important role in the prevention of spread. The Study to Optimize Peritoneal Infection Therapy (STOP-IT) trial has challenged the previous paradigm of length of antibiotic treatment whereas biomarkers such as procalcitonin are playing a prominent role in individualizing therapy. Several novel therapies for refractory septic shock, while still investigational, are gaining prominence rapidly (such as vitamin C) whereas others await further clinical trials. Management strategies presented as care bundles continue to be updated by the Surviving Sepsis Campaign, yet still remain controversial in its global adoption. We have broadened our temporal and epidemiologic perspective of sepsis by understanding it both as an acute, time-sensitive, life-threatening illness to a chronic condition that increases the risk of mortality up to five years post-discharge. Artificial intelligence, machine learning, and bedside scoring systems can assist the clinician in predicting post-operative sepsis. The public health role of the surgeon is key. This includes collaboration and multi-disciplinary antibiotic stewardship at a hospital level. It also requires controlling pharmaceutical sales and the unregulated dispensing of antibiotic agents globally through policy initiatives to control emerging resistance through prevention.
\end{abstract}

Keywords: MDROs; procalcitonin; sepsis; source control; vitamin C

$\mathbf{S}$ EPSIS HAS BEEN RECOGNIZED INCREASINGLY as a potentially life-threatening condition that merits the same respect as acute myocardial infarctions, acute ischemic strokes, or other such time-sensitive pathologies recognized in the emergency department, hospital ward, or critical care unit. There have been multiple updates in the management of sepsis since the first definition of sepsis was published in 1992. Some have persisted in its strength of evidence, whereas others have been disproven or even demonstrated harm. Drotrecogin alfa (activated) is an example of one such intervention that was adopted broadly through society guidelines worldwide, sponsored by industry [1], yet found to cause increased harm to patients, prompting voluntary removal from the market in 2011 [2]. Conversely, the ongoing strength of evidence for the early administration of antibiotic agents remains solid [3]. Areas of controversy remain, however, with the duration of antibiotic therapy with large, randomized control trials demonstrating the non-inferiority

\footnotetext{
${ }^{1}$ Division of Trauma, Critical Care, Burns, and Acute Care Surgery, MetroHealth Medical Center, Cleveland, Ohio.

${ }^{2}$ Trauma, Emergency Surgery and Surgical Critical Care, Harvard Medical School, Boston, Massachusetts.

${ }^{3}$ Division of Trauma and Surgical Critical Care, University of Miami Miller School of Medicine, Miami, Florida.

${ }^{4}$ Division of General \& Acute Care Surgery, Medical University of South Carolina, Charleston, South Carolina.

${ }^{5}$ Section for Trauma and Acute Care Surgery, The University of Chicago Medicine, Chicago, Illinois.
} 
of a shorter course compared to our historic longer courses [4]. Other interventions have had varying evidence in support of or against steroids, type of fluids used for resuscitation, or clear end points or goals of resuscitation. As new interventions for sepsis continue to emerge, these have been organized into sepsis care bundles by society guidelines to assist the clinician in the rational application of such therapies. External validity in real-world application of evidence-based bundles is low with a wide variation in practice patterns of sepsis management remaining a challenge worldwide [5].

This is important globally because of emerging multidrug-resistant organisms (MDROs), the use and abuse of antibiotic agents, and challenges in achieving adequate source control. Antibiotic stewardship in hospitals remains a public health challenge on a grand scale that can only be approached in a multidisciplinary, collaborative fashion. The importance of organizing a rapid diagnostic strategy and institutional approach to antimicrobial stewardship is a key local component to controlling the spread of MDROs. Surgeons have a key role both in extirpative sepsis management as well as intensivists through our early recognition in vulnerable or critically ill patients. Our role as policy leaders also includes examining the evidence-based literature to support modern approaches to institutional pathways for sepsis detection, treatment and controlling the spread of future infections.

The purpose of this review is to discuss the modern advances and controversies regarding the diagnosis and treatment of sepsis, as well as discuss the upcoming challenges and remaining research priorities for future advancement in the science of sepsis. We aim to emphasize the important role that surgeons encompass in the treatment of patients with severe surgical infections.

\section{Sepsis Definitions}

The term sepsis originates from an ancient Greek word sepsis, which means the "decay of organic matter," and was first known to be used in an Egyptian papyrus circa 1600 B.C. [6]. The modern definition of sepsis was created in 1991 by a consensus conference of the American College of Chest Physicians (ACCP) and the Society of Critical Care Medicine (SCCM), so that clinicians could apply a standard framework with precise diagnostic guidelines to follow epidemiologic trends and determine efficacy of treatments for sepsis $[7,8]$. The conference defined a new term, the systemic inflammatory response syndrome (SIRS), based on a clinical constellation of abnormal temperature, heart rate, respiratory rate, and white blood cell count. Sepsis was defined as the presence of SIRS with an infection present. Severe sepsis was defined as sepsis with organ dysfunction, and septic shock was sepsis-induced hypotension despite adequate resuscitation. These definitions were updated in 2003 (Sepsis-2) but the definition remained largely unchanged [9].

In 2016, a task force was convened between the SCCM and the European Society of Intensive Care Medicine (ESICM) to revise the definitions of sepsis [10,11]. For the first time in more than 20 years, the definitions of sepsis changed considerably [12]. The panel was concerned that the prior definition lacked specificity to truly predict mortality and performed not only a review of existing literature but also cohort studies using registries from the Surviving Sepsis Campaign and electronic health records to identify models of sepsis that provided improved prediction of sepsis-associated mortality. These definitions are known as Sepsis-3.

Sepsis-3 redefined sepsis as "life-threatening organ dysfunction caused by a dysregulated host response to infection." Septic shock was defined as "a subset of sepsis in which underlying circulatory, cellular, and metabolic abnormalities are associated with a greater risk of mortality than sepsis alone." Septic shock requires the clinical criteria of sepsis plus the need for vasopressor therapy to maintain mean arterial pressure above $65 \mathrm{~mm} \mathrm{Hg}$, and a lactate $>2 \mathrm{mmol} / \mathrm{L}$ despite adequate fluid resuscitation. Systemic inflammatory response syndrome was removed from the definition because of high rates of false-positives and poor discrimination ability [11]. The terms SIRS and severe sepsis were dropped. By Sepsis-3, sepsis became a more severe disease with higher mortality than previously defined; studies comparing Sepsis3 definitions have confirmed that the reclassification of patients is associated with higher mortality than the prior definition [13-15].

For the diagnosis of sepsis, the Sequential Organ Failure Assessment (SOFA) score could be used, but this is not used routinely at the bedside. To aid clinicians, the Sepsis-3 authors created a new score, the quick Sequential Organ Failure Assessment (qSOFA) score. Their goal was to create a bedside diagnostic score with reasonable predictive validity for sepsis-related in-hospital mortality and prolonged intensive care unit (ICU) stay. To create this score, the authors utilized a population of critically ill patients with known or suspected infection. The outcome of interest utilized in their scoring model was increased risk of death or a prolonged ICU stay. The qSOFA score is based on three criteria: altered mental status, fast respiratory rate, and low blood pressure (area under the receiver operating characteristic curve [AUROC] 0.81 , confidence interval [CI] $0.80-0.82$ ) [16]. This tool is best used as a prognostic tool for mortality in patients with sepsis, not as a diagnostic tool to rule out the presence of sepsis (Table 1).

As a result of these changes, the new Sepsis-3 definitions [17] have been the source of thoughtful debate and controversy, especially regarding the importance of early identification and diagnosis of infection. As described above, because the qSOFA was not designed to identify infection early, one valid argument against these criteria theorizes that application of these new criteria may lead to a failure to recognize the signs of potentially lethal infection until the outcomes are poor. In addition, these new criteria were derived from patients with suspected infection, so there is no consideration as to how to exclude patients with inflammatory states that are the result of non-infectious causes. Since the new definitions, several studies have been performed questioning the usefulness of qSOFA as a screening tool for the early diagnosis of sepsis. These studies suggest that SIRS has a higher sensitivity, whereas qSOFA has a higher specificity for poor outcomes. It may make sense to continue to apply SIRS criteria in the earliest stages of infection, although there continues to be no consensus on this important question.

To further complicate matters, the Centers for Medicare and Medicaid Services (CMS) has an alternate definition for sepsis and septic shock that continues to use the prior definitions, plus the use of specific end-organ dysfunction criteria and lactate criteria [18]. Whereas the cost and reimbursement 
TABle 1. REVised Definitions of SePSis

\begin{tabular}{|c|c|c|}
\hline & 1992 Consensus Statement [7] & Sepsis-3 [11] \\
\hline SIRS & $\begin{array}{l}\text { Four Criteria of SIRS } \\
\text { Temperature }>38^{\circ} \mathrm{C} \text { or }<36^{\circ} \mathrm{C} \\
\text { Heart rate }>90 \text { beats per minute } \\
\text { Respiratory rate }>20 \text { breaths per minute } \\
\text { or } \mathrm{PaCO}_{2}<32 \mathrm{~mm} \mathrm{Hg} \\
\text { White blood cell count }>12,000 / \mathrm{cu} \mathrm{mm} \text {, } \\
<4,000 / \mathrm{cu} \text { mm or } 10 \% \text { bands }\end{array}$ & Eliminated \\
\hline Sepsis & $\begin{array}{l}\text { Systemic response to infection, manifested } \\
\text { by two or more SIRS criteria }\end{array}$ & $\begin{array}{l}\text { Life-threatening organ dysfunction caused by a } \\
\text { dysregulated host response to infection. Organ } \\
\text { dysfunction defined as an increase in } 2 \text { points or } \\
\text { more in the SOFA score, which includes respi- } \\
\text { ration, coagulation, liver function, hypotension, } \\
\text { altered mental status, and kidney dysfunction. } \\
\text { ICU patients with increased risk of mortality or } \\
\text { prolonged ICU stay can be identified with a } \\
\text { qSOFA score }>2 \text { : } \\
\text { Altered mental status; Glasgow Coma Scale }<15 \\
\text { Respiratory rate }>22 \text { breaths per minute } \\
\text { Systolic blood pressure }<100 \mathrm{~mm} \mathrm{Hg}\end{array}$ \\
\hline Severe sepsis & $\begin{array}{l}\text { Sepsis associated with organ dysfunction, } \\
\text { hypoperfusion, or hypotension. Hypoperfusion } \\
\text { and perfusion abnormalities may include, but } \\
\text { are not limited to lactic acidosis, oliguria, or an } \\
\text { acute alteration in mental status }\end{array}$ & Eliminated \\
\hline Septic shock & $\begin{array}{l}\text { Sepsis-induced hypotension despite adequate } \\
\text { fluid resuscitation, along with the presence of } \\
\text { perfusion abnormalities that may include, but } \\
\text { are not limited to, lactic acidosis, oliguria, or } \\
\text { an acute alteration in mental status. Patients } \\
\text { who are receiving inotropic or vasopressor } \\
\text { agents may not be hypotensive at the time that } \\
\text { perfusion abnormalities are measured }\end{array}$ & $\begin{array}{l}\text { Subset of sepsis in which underlying circulatory } \\
\text { and cellular/metabolic abnormalities are pro- } \\
\text { found enough to substantially increase mortality. } \\
\text { Criteria: } \\
\text { Sustained systolic blood pressure }<90 \mathrm{~mm} \mathrm{Hg} \\
\text { requiring vasopressors to maintain mean arterial } \\
\text { pressure }>65 \mathrm{~mm} \mathrm{Hg} \text {, and } \\
\text { Lactate }>2 \mathrm{mmol} / \mathrm{L} \text {, despite adequate volume } \\
\text { resuscitation }\end{array}$ \\
\hline
\end{tabular}

SIRS = systemic inflammatory response syndrome; $\mathrm{PaCO}_{2=}$ partial pressure of carbon dioxide $;$ SOFA= Sequential [Sepsis-related] Organ Failure Assessment; qSOFA=Quick Sequential Organ Failure Assessment; ICU = intensive care unit;

implications of these discrepancies are outside of the scope of this review, the use of multiple definitions of sepsis will likely have far-reaching clinical, research, administrative, and economic impact.

\section{Source Control, Antibiotic Choice, MDROs}

It is important for the acute care surgeon to consider the rapid emergence of MDROs, source control, and applying the principles of both under a framework of public health prevention. Often surgeons are faced with a variety of clinical scenarios involving vulnerable patient populations: the elderly patient with diverticulitis with dislodged percutaneous abdominal drains to the intubated patient with flank necrotizing soft tissue infection from a missed perforated retrocecal appendix. Often, we are called upon to administer the appropriate antibiotic agents, achieve adequate source control, and do both in a timely fashion for improved clinical outcomes. Multi-drug-resistant organism infections are concerning because these occur in the weakest hosts with the most virulent bacteria, increasing mortality. Therapy may be delayed or inadequate, spread may be rapid, and there are limited antibiotic options. The challenge in initiating the appropriate antibiotic regimen is that many of our patients present with risk factors for MDROs and our usual empiric antibiotic coverage is suboptimal against these organisms. Additionally, there may be challenges in achieving adequate source control, leading to delays in therapy that have consequences for patient outcomes. Antibiotics and source control are both important and complementary therapeutic modalities for the acute care surgeon, whose decisions must follow antibiotic stewardship principles to limit the spread of MDROs.

In February 2017, the World Health Organization released a priority pathogen list, calling for urgent development of new antimicrobials for these [19]. The focus was on gramnegative bacteria with carbapenem resistance. Resistance can be acquired, typically through horizontal transfer of plasmids, leading to MDROs, but can be intrinsic or adaptive as well [20]. Whereas multi-drug resistance is defined as non-susceptibility to one agent in three or more antimicrobial categories, extensive and pan-drug resistance is also an emerging problem [21].

Clinical breakpoints are important to understand, as the minimum inhibitory concentration (MIC) of a drug needed for activity against a bacterium may depend on the tissue targeted/route of drug administration, mechanism of bacterial resistance, and if specific drug combinations will be used 
synergistically. The MIC breakpoints may also vary globally because these are determined somewhat arbitrarily by different global bodies such as European Committee for Antimicrobial Susceptibility Testing (EUCAST), Clinical \& Laboratory Standards Institute (CLSI), or the U.S. Food and Drug Administration [22]. Priorities rest on identifying patients who are at risk of MDROs when they present to the acute care surgeon with a complicated intra-abdominal infection (cIAI). Risk factors include hospital-acquired cIAI, such as anastomotic leaks, antimicrobial therapy in the previous seven days, leukocytosis or leukopenia, severe cardiovascular disease, and inadequate source control [23]. If an MDRO is suspected, standard empiric therapy, based on one's hospital's antibiogram, may be inadequate. Detection of MDROs can be facilitated with rapid identification methods (RIMs), molecular-based techniques to identify concerning organisms such as methicillin-resistant Staphylococcus areus (MRSA), vancomycin-resistant enterococcus (VRE), or carbapenem-resistant Enterobacteriaceae (CREs) as early as two hours after a specimen is collected, allowing the clinician to escalate or de-escalate as needed. A key component to RIMs is that the results must be communicated immediately to the clinician by the laboratory [24].

The classic ESKCAPE (Enterococcus faecium, Staphylococcus aureus, Klebsiella pneumoniae, Acinetobacter baumannii, Pseudomonas aeruginosa, and Enterobacter species) organisms are the common, nosocomial gram-positive and gram-negative MDROs that include Acinetobacter baumannii and Pseudomonas aeruginosa, both resistant to carbapenems. Increasing concerns of Clostridium difficile resistance have broadened the acronym [25]. There is also growing resistance of VRE to linezolid, with several new promising glycopeptides. A novel fifth-generation cephalosporin ceftaroline has excellent activity against MRSA [26]. For the CREs in cIAI, ceftazidime-avibactam as monotherapy is inadequate $[27,28]$ but may be used in combination with metronidazole for anaerobic coverage in cIAI and is active against extended-spectrum beta-lactamase (ESBL) gram-negatives. Similarly, ceftolazone-tazobactam is another newer beta-lactam-beta lactamase inhibitor combination (BLI), which again requires additional anaerobic coverage for cIAI [29]. This drug is first-line therapy for MDR Pseudomonas aeruginosa. Carbapenem-BLI combinations (meropenem-vaborbactam) are also available for MDRO infections [30]. Eravacycline is a new tetracycline of the fluorocycline subgroup that is active against a wide spectrum of gram-positive and gram-negative pathogens (MRSA, VRE, and Enterobacteriaceae with ESBLs), although it does not have activity against Pseudomonas aeruginosa. As always, acute care surgeons have a responsibility to be leaders in local antibiotic stewardship programs that limit the overuse of antibiotic agents and mandate handwashing for all. Multidisciplinary communication, involving infectious diseases and pharmacy, is a key component of patient care.

It is incumbent on the acute care surgeon to consider the risk factors for MDROs for newly presenting patients, especially those with hospital-acquired infections. After starting appropriate empiric antibiotic agents based on risk factors and your hospital's antibiogram, consider rapid molecular testing to rule MDROs in or out expeditiously. Always remember to consider an underlying focus of pus, necrotic tissue, or combination thereof anywhere in the body that needs source control. Procalcitonin can be used as a marker of resolution of sepsis to judge the adequacy of drainage, debridement or need for re-laparotomy [31,32]. Surgeons together with their infectious disease colleagues have a key role in limiting the spread of MDROs in their institutions, with preventive measures being essential.

\section{Duration of Antibiotic Agents and Biomarkers}

A randomized controlled trial known as the Study to Optimize Peritoneal Infection Therapy (STOP-IT) compared antibiotic duration of four days to a longer course with cessation two days after the resolution of clinical symptoms, for a maximum of 10 days. Antimicrobial duration was measured from the patient's index source-control procedure, which could include surgery or drainage. The authors found that there was no difference in outcomes, especially mortality, when using the short course [1]. On subgroup analysis, a fourday course of antibiotic agents was also safe with certain risk factors (such as diabetes or obesity) [33], regardless of source control method [34] and even in severe illness (sepsis, high APACHE II) [35]. Caveats to these findings include the need for source control, regardless of method used, and that these results cannot be applied to immunocompromised patients, who remain understudied. Prior to the publication of STOP-IT, the Surviving Sepsis Campaign (SSC) guidelines recommended seven to 10 days of antibiotic coverage as a starting point based on limited data of low quality.

The recommendations stipulate that this can be shortened when dealing with genitourinary infections or cIAI with adequate source control. Longer courses are recommended when there is inadequate source control, the presence of some MDROs, Staphylococcus aureus bacteremia, an immunosuppressed host, and with some fungal or viral infections. Precisely what shorter or longer implies remains unclear. The SSC guidelines regarding cIAI need to be updated in light of the STOP-IT trial.

Procalcitonin is demonstrating promise in individualizing antibiotic use. Evidence shows that this proinflammatory biomarker safely shortens antibiotic duration in critically ill patients in the ICU [36,37], and has been demonstrated to reduce mortality [38] in these and other patients. In a recent Cochrane Review, the use of procalcitonin to guide initiation and duration of antibiotic treatment resulted in lower risks of mortality, lower antibiotic consumption, and lower risk for antibiotic-related side effects [39]. Further studies are needed to determine if procalcitonin can safely guide clinicians to utilize ultra-short courses of antibiotic agents. Other biomarkers are currently under investigation. For example, reduced monocyte human leukocyte antigen-DR (HLA-DR) expression as measured by flow cytometry is a novel biomarker in sepsis and provides valuable information in terms of predicting mortality and risk of secondary infections [40]. Circulating DNA (cell-free DNA) is released from a number of cells, including neutrophils, eosinophils, and macrophages, as a result of either apoptosis or other forms of cellular damage and can serve as an early biomarker in sepsis [41]. A new metagenomics-based test that detects microbial DNA in human blood can identify a diverse array of pathogens from any source in the body and be used similar to biomarkers for the early identification of sepsis-inducing pathogens [42]. 


\section{Other Novel Therapies in Refractory Septic Shock: Vitamin C, Thiamine, Angiotensin II, and Methylene Blue}

The following treatments for septic shock may be useful in refractory or vasoplegic septic shock. However, all therapies discussed in this section are investigational with limited evidence. Careful appraisal of the literature and its application to their patient should be undertaken by clinicians.

Vitamin $\mathrm{C}$ is known for its antioxidant and antiinflammatory [43] properties in the human body, characteristics that make this a favorable adjunct to use in sepsis. Vitamin $\mathrm{C}$ is also required to produce catecholamines [44] and is synergistic with thiamine and glucocorticoids [45], both helpful in sepsis. Interestingly, $100 \%$ of patients with sepsis have low vitamin C levels [46] possibly because of intrinsic depletion of endogenous levels during the septic state. Limited clinical data of 146 patients from three studies, including two randomized controlled trials, [47-49] demonstrated a large mortality effect (odds ratio [OR] 0.17 [0.070.40 ], $\mathrm{p}<0.001$ ) [50] with the administration of high doses of vitamin $\mathrm{C}$ during refractory septic shock. There were also nearly no adverse effects with vitamin $\mathrm{C}$ in these critically ill patients [45]. One recently published protocol used intravenous vitamin C $1.5 \mathrm{~g}$ every six hours for four days coupled with intravenous thiamine $200 \mathrm{mg}$ every 12 hours for four days [49]. A tapered, seven-day course of intravenous hydrocortisone $50 \mathrm{mg}$ every six hours has also been included in these studies, and may be an important confounder [51]. Currently, there are 13 trials ongoing investigating the beneficial properties of high-dose vitamin $\mathrm{C}$ in refractory septic shock. Angiotensin II has also been advocated by some, as limited clinical data $(n=344)$ have demonstrated an improved mean arterial pressure (MAP) in refractory shock and improved cardiovascular SOFA scores at 48 hours when administered at $20 \mathrm{ng} / \mathrm{kg} / \mathrm{min}$ (maximum, 200) to MAP goal [52]. Clinicians need to exercise caution in patients with cardiac issues given its potential for vasoconstriction.

Methylene blue is known for its inhibition of the production of nitric oxide (NO) [53], which is increased in septic shock [54]. Excessive NO leads to persistent vasodilation [55] and thus is another driver of refractory septic shock. Again, there exist only limited clinical data $(n=114,11$ studies, two randomized controlled trials) [56] that have demonstrated that methylene blue can improve MAP for two to three hours using a dose of $1-2 \mathrm{mg} / \mathrm{kg}$ for $10-15$ minutes. It currently remains unknown if bolus or infusion of methylene blue is more favorable for MAP response and overall outcomes. Methylene blue will render pulse oximetry data unreliable and is contraindicated in patients with glucose-6phosphate-dehydrogrenase deficiency. Given the propensity for pulmonary vasoconstriction, extreme caution must be exercised in patients with pneumonia, pulmonary hypertension, or acute respiratory distress syndrome (ARDS) as a cause or result of sepsis.

Other novel therapies include polyclonal or monoclonal antibodies for sepsis, with polyclonal intravenous immunoglobulin (IVIG) demonstrating a mortality reduction, but only in clinical trials at risk of bias, in an updated Cochrane Review [57]. Beta-blockade, in particular with esmolol, has demonstrated promise in several randomized controlled trials, including demonstrating a reduction in morality from sepsis [58]. Extracorporeal blood purification strategies are currently under investigation, as are industry-sponsored adsorptive membranes, as adjuvant therapy for sepsis [59]. Similarly, therapeutic plasma exchange has been investigated for similar reasons with some promising data [60]. Additional clinical studies are needed to clarify which novel therapies will be beneficial for the treatment of sepsis and septic shock.

\section{Surviving Sepsis Campaign}

Guidelines for the management of severe sepsis and septic shock by the SSC initially were published in 2004 and were followed by updates in 2008 and 2012 [61,62]. The SSC, initiated in 2002, was designed to reduce mortality and has provided key guidance to the medical community for these endeavors. The SSC guidelines provide evidence-based guidance for treatment based on all major physiologic categories affected by sepsis, including initial resuscitation, screening and performance improvement, diagnosis, use of antimicrobials, fluids, and vasopressors, and source control. In 2014, a 7.5-year longitudinal study showed that compliance with bundles based on the guidelines from 2004 showed a $25 \%$ risk reduction in mortality rate; each $10 \%$ increase in compliance and additional quarter of participation with the guidelines was associated with a decrease in odds ratio for hospital mortality [64]. Despite overall low compliance in an international study, the International Multicentre Prevalence Study on Sepsis (IMPreSS) study [51], also showed that higher levels of compliance reduced mortality from sepsis.

One new recommendation from the SSC guidelines from 2016 deserves particular mention for the modern care of the septic patient. The 2016 SSC guidelines suggest the use of "dynamic resuscitation markers" to determine fluid responsiveness, whereas recommendations from 2012 suggested use of central venous oxygen saturation to guide goaldirected therapy. Once initial resuscitation of $30 \mathrm{~mL} / \mathrm{kg}$ is complete, fluid administration should be judiciously administered only for patients who remain fluid responsive. In 2016, a review of the literature concluded that the use of CVP alone can no longer be justified because of poor identification of fluid responsiveness [65]. Multiple trials showed no benefit to early goal-directed therapy for sepsis [66-68]. In place of CVP and central venous oxygen saturation (ScVO2) measurement, dynamic assessment with use of the passive leg raise or bedside ultrasound may provide a more accurate determination of fluid responsiveness. In other words, before administering large fluid volumes, a patient should demonstrate an increased stroke volume with an increase in preload, to ensure that the patient continues to be on the ascending limb of the Frank-Starling curve and prevent iatrogenic fluid overload. Whereas the optimal method to measure fluid responsiveness remains unknown [70-72], current European guidelines recommend the early and repeat use of echocardiography in shock to guide fluid responsiveness. Transpulmonary thermodilution is also useful as an individualized approach to the complex critical care patient in shock, in particular with concomitant ARDS, with pulmonary artery catheters not recommended for routine use [73].

The most recent SSC guidelines were succeeded by publication of a one-hour bundle, published in 2018, that recommends the following bundle of care: (1) measure lactate level; remeasure if initial lactate is $>2 \mathrm{mmol} / \mathrm{L}$; (2) obtain 
blood cultures prior to administration of antibiotic agents; (3) administer broad-spectrum antibiotic agents; (4) begin rapid administration of $30 \mathrm{~mL} / \mathrm{kg}$ crystalloid for hypotension or lactate $\geq 4 \mathrm{mmol} / \mathrm{L}$; (5) apply vasopressors if patient is hypotensive during or after fluid resuscitation to maintain MAP $\geq 65 \mathrm{~mm} \mathrm{Hg}$ [74]. This is a change from the prior bundles published by the SSC, which were based on administration of treatment within three or six hours, to encourage clinicians to diagnose sepsis quickly and begin treatment immediately.

This SSC one-hour bundle has generated controversy, debate [75], and criticism because of a lack of clear evidence, the potential for indiscriminate antibiotic agent use, and the presumptiveness of the SSC to dictate healthcare policy and the behavior of thousands of physicians without adequate basis to do so. This prompted a joint statement by the SCCM and the American College of Emergency Physicians (ACEP) that recommends against the one-hour bundle [76]. An online, international petition has collected more than 6,000 signatures calling for the retirement of these updated SSC guidelines due, in part, to the global monopoly they hold as well as industry influence $[1,77]$. However, leaders in the field are instead recommending the generation of multiple sepsis guidelines from various countries as "competing sepsis guidelines could promote a diversity of opinions, regional adaptation, and flexible thinking about different approaches to sepsis" [78].

\section{Sepsis Outcomes and Predicting Sepsis}

\section{In-hospital mortality}

The mortality of sepsis is reported to range between approximately $10 \%$ and $52 \%$ [6]. In a study by Martin et al. [79] that included more than 10 million patients between 1979 and 2000, the in-hospital mortality of patients with sepsis was shown to have been decreasing from a high of more than $30 \%$ in the early 1980s to an average of $17.9 \%$ in the last six years. Similarly, Dombrovskiy et al. [80] showed that the number of hospitalizations and total mortality from sepsis in the United States have increased between 1993 and 2003, but the case fatality rate has decreased. The mortality of sepsis increases as its severity increases. Rangel-Frausto et al. [81] elegantly demonstrated that the mortality rate of patients increased incrementally from $16 \%$ to $20 \%$ and $46 \%$ as they moved from sepsis to severe sepsis and then septic shock.

\section{Long-term mortality}

Several studies have attempted to examine the long-term post-discharge survival of sepsis patients. Nesseler et al. [82] suggested that the six-month mortality rate of patients with sepsis is as high as $45 \%$. Other studies suggested that these patients, even when they survive, continue to have subsequent serious infections and increased healthcare utilization more than a year later $[83,84]$. In a nationwide populationbased study, Ou et al. [85] suggested that sepsis survivors are at increased risk for major cardiovascular events and allcause mortality at one-year post-discharge, and that that risk remains elevated up to five years later.

\section{Predicting the outcome of sepsis}

The results of studies examining the ability of qSOFA and SOFA scores in predicting outcome of patients with sepsis have been conflicting. Although qSOFA has been suggested to be superior in predicting mortality compared with SIRS, sepsis, and severe sepsis criteria [86], its ability to predict mortality remains questionable $[87,88]$. A recent study by Raith et al. [89] showed the significant superiority of SOFA to qSOFA in predicting the outcome of septic patients.

\section{Predicting sepsis}

Whereas qSOFA could be useful to prognosticate patients with sepsis and predict their risk of mortality, it is not recommended as a sepsis screening tool. Haydar et al. [90] warned that qSOFA alone more than doubles the time to identify sepsis in the emergency room compared with SIRS screening criteria and may even delay the initiation of therapy for sepsis, thus worsening outcomes. In the emergency surgery patient, the Emergency Surgery Score (ESS) calculated pre-operatively has been shown to predict post-operative outcome accurately including post-operative sepsis (Table 2) [91-93]. In the last few years, the use of artificial intelligence has been promising. For example, POTTER interactive and user-friendly calculator, available for download on smartphones, used machine learning techniques to train complex algorithms to predict outcome in the high-risk emergency surgery patient. Specifically, because of the non-linear nature of its algorithm, its accuracy in predicting postoperative

Table 2. The Emergency Surgery Score

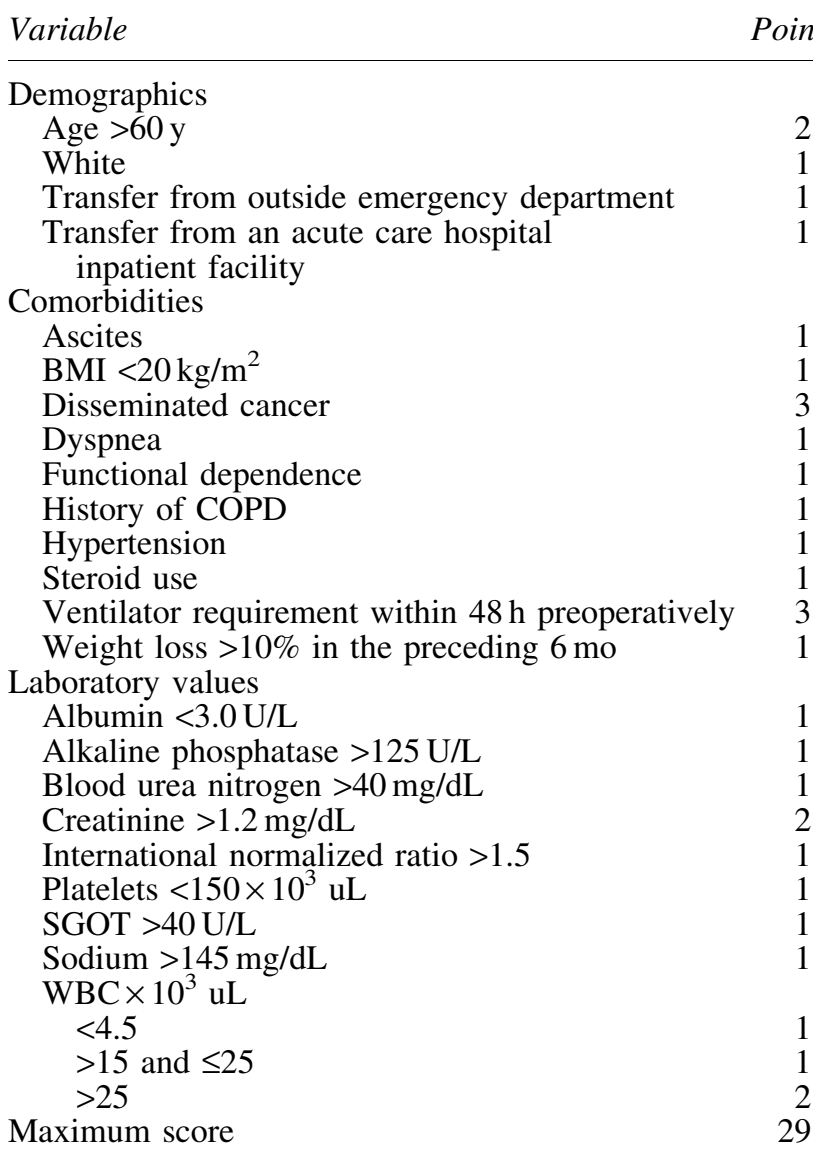

$\mathrm{BMI}=$ bone mineral density $\mathrm{COPD}=$ chronic obstructive pulmonary disease; $\mathrm{SGOT}=$ serum glutamic oxaloacetic transaminase; $\mathrm{WBC}=$ white blood cell count . 


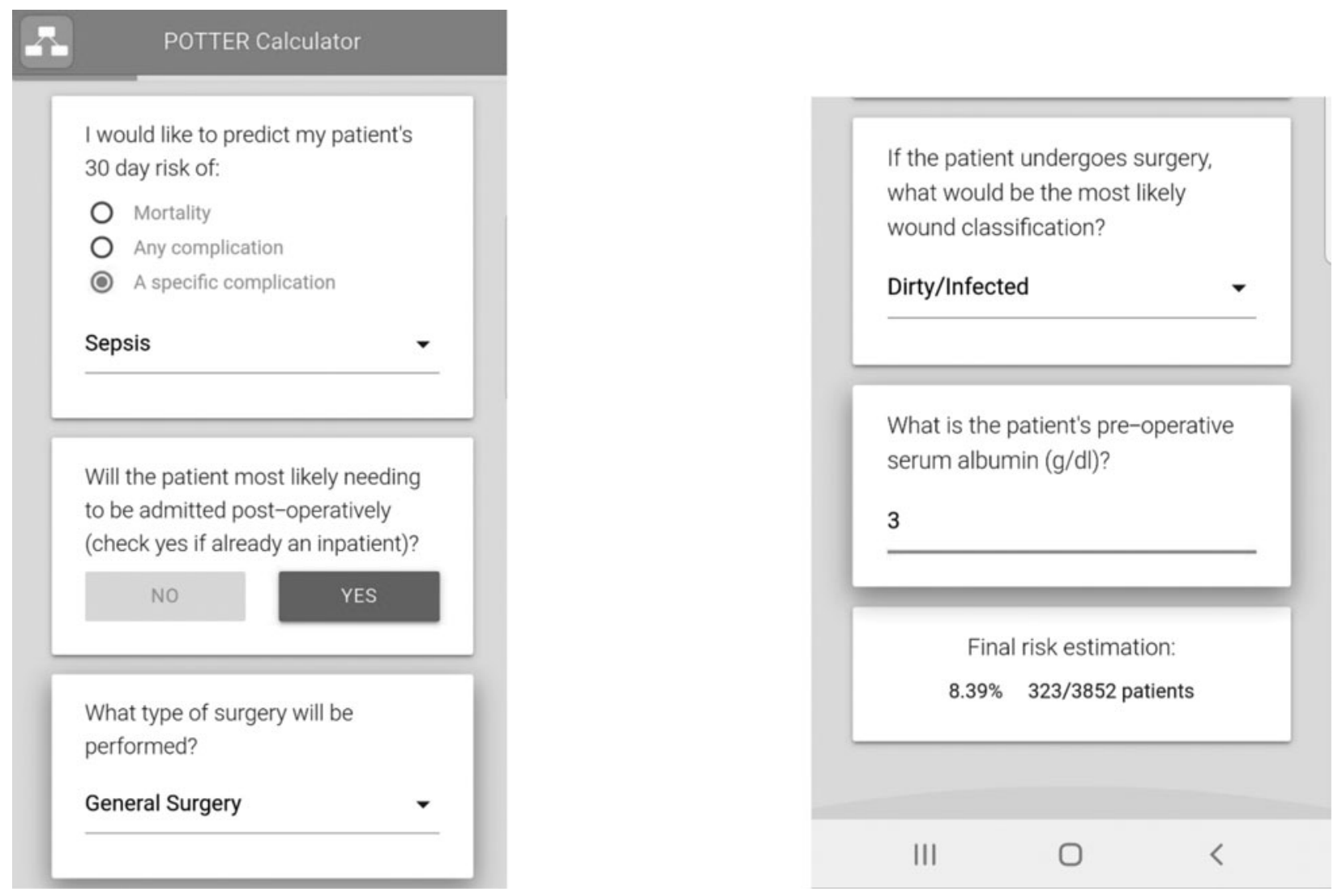

FIG. 1. An example illustrating the ability of the artificial intelligence based and interactive POTTER phone application to predict post-operative sepsis in a highly accurate fashion (AUROC 0.93).

sepsis has been shown to be several folds superior in this patient population [95].

In addition to POTTER, machine learning techniques have been used to analyze vital signs and heart rate variability in real time to predict patients in early sepsis [96-101]. For example, the machine learning algorithm Insight was derived from six vital signs and outperformed existing scoring systems for sepsis and septic shock [99]. Such algorithms promise, once integrated into electronic health records, to trigger rescue alerts identifying the clinically deteriorating patient with sepsis who has not yet shown overt signs of sepsis. Such triggers may help mitigate the organ system deleterious effects of the delayed recognition of sepsis.

\section{Future Areas for Research/Conclusion}

In summary, the definitions, diagnosis, and management of sepsis continue to evolve. Although imperfect, Sepsis-3 and evaluation by qSOFA are the new lingua franca if surgeons, intensivists, infectious disease physicians, and other medical specialists are to make coordinated progress in the field. Source control remains the primary and critical responsibility of surgeons. However, the inability to obtain definitive source control remains a real problem in many clinical scenarios and commonly leads to protracted courses of antibiotics. Antibiotic stewardship is complicated when source control cannot be obtained, and there is a paucity of data regarding duration of antibiotics in these situations. More research is needed to determine antibiotic choice and duration if source control is unattainable. The complexity of the mechanisms of resistance and the speed at which resistance is emerging require that surgeons cooperate with infectious disease and pharmacy specialists. The mass of information on new antibiotics and diagnostic methods demands multi-disciplinary cooperation. When prevention fails and we must treat sepsis, novel and inexpensive therapies, such as vitamin $\mathrm{C}$, thiamine, hydrocortisone, methylene blue, and similar non-commercial therapies should be used to the extent the literature supports them. Further research is needed but is unlikely to be done if not publicly funded. Last, we must look beyond our patients and consider a broader approach to sepsis and antimicrobial resistance. It will be imperative to employ a global public health approach, as the wide availability of over-the-counter antibiotic agents in much of the world exacerbates resistance patterns. We must also look outside our own sphere of influence. The use of antibiotic agents and antifungals in animal agriculture and farming dwarfs the use of these agents in human disease, producing new mechanisms of resistance and this has to be addressed at a level higher than our medical professional societies. Epidemiologic research in the outcomes of sepsis, after hospitalization ends, casts a light on the gravity of the problem of sepsis and the need for ongoing basic and clinical research in the years to come.

\section{Acknowledgments}

Presented at the American College of Surgeons' Clinical Congress of 2018. 


\section{Funding Information}

No funding was received for this article.

\section{Author Disclosure Statement}

No competing financial interests exist.

\section{References}

1. Eichacker PQ, Natanson C, Danner RL. Surviving sepsis-Practice guidelines, marketing campaigns, and Eli Lilly. N Engl J Med 2006;355:1640-1642.

2. FDA Drug Safety Communication: Voluntary market withdrawal of Xigris [drotrecogin alfa (activated)] due to failure to show a survival benefit. FDA [Internet]. 2018 Nov 3 [cited 2019 May 7]; Available from: /drugs/drugsafety-and-availability/fda-drug-safety-communicationvoluntary-market-withdrawal-xigris-drotrecogin-alfaactivated-due (last accessed November 15, 2019).

3. Sherwin R, Winters ME, Vilke GM, Wardi G. Does early and appropriate antibiotic administration improve mortality in emergency department patients with severe sepsis or septic shock? J Emerg Med 2017;53:588-595.

4. Sawyer RG, Claridge JA, Nathens AB, et al. Trial of short-course antimicrobial therapy for intraabdominal infection. N Engl J Med 2015;372:1996-2005.

5. Jozwiak M, Monnet X, Teboul J-L. Implementing sepsis bundles. Ann Transl Med 2016;4:332.

6. Kempker JA, Martin GS. The changing epidemiology and definitions of sepsis. Clin Chest Med 2016;37:165-179.

7. Bone RC, Balk RA, Cerra FB, et al. Definitions for sepsis and organ failure and guidelines for the use of innovative therapies in sepsis. The ACCP/SCCM Consensus Conference Committee. American College of Chest Physicians/Society of Critical Care Medicine. Chest 1992;101: 1644-1655.

8. American College of Chest Physicians/Society of Critical Care Medicine Consensus Conference: Definitions for sepsis and organ failure and guidelines for the use of innovative therapies in sepsis. Crit Care Med 1992;20:864-874.

9. Levy MM, Fink MP, Marshall JC, et al. 2001 SCCM/ ESICM/ACCP/ATS/SIS International Sepsis Definitions Conference. Crit Care Med 2003;31:1250-1256.

10. Shankar-Hari M, Phillips GS, Levy ML, et al. Developing a new definition and assessing new clinical criteria for septic shock: For the Third International Consensus Definitions for Sepsis and Septic Shock (Sepsis-3). JAMA 2016;315:775-787.

11. Singer M, Deutschman CS, Seymour CW, et al. The Third International Consensus Definitions for Sepsis and Septic Shock (Sepsis-3). JAMA 2016;315:801-810.

12. Napolitano LM. Sepsis 2018: Definitions and guideline changes. Surg Infect 2018;19:117-125.

13. Butcher A, Richards T. Cornerstones of patient blood management in surgery. Transfus Med Oxf Engl 2018;28: 150-157.

14. Fernando SM, Reardon PM, Rochwerg B, et al. Sepsis-3 septic shock criteria and associated mortality among infected hospitalized patients assessed by a rapid response team. Chest 2018;154:309-316.

15. Demiselle J, Wepler M, Hartmann C, et al. Hyperoxia toxicity in septic shock patients according to the Sepsis-3 criteria: A post hoc analysis of the HYPER2S trial. Ann Intensive Care 2018;8:90.
16. Seymour CW, Liu VX, Iwashyna TJ, et al. Assessment of clinical criteria for sepsis: For the Third International Consensus Definitions for Sepsis and Septic Shock (Sepsis-3). JAMA 2016;315:762-774.

17. Marshall JC. Sepsis-3: What is the meaning of a definition? Crit Care Med 2016;44:1459-1460.

18. Kalantari A, Mallemat H, Weingart SD. Sepsis definitions: The search for gold and what CMS got wrong. West J Emerg Med 2017;18:951-956.

19. World Health Organization. WHO publishes list of bacteria for which new antibiotics are urgently needed. ization. www.who.int/news-room/detail/27-02-2017-whopublishes-list-of-bacteria-for-which-new-antibiotics-areurgently-needed (Last accessed October 28, 2018).

20. Arzanlou M, Chai WC, Venter H. Intrinsic, adaptive and acquired antimicrobial resistance in gram-negative bacteria. Essays Biochem 2017;61:49-59.

21. Magiorakos A-P, Srinivasan A, Carey RB, et al. Multidrugresistant, extensively drug-resistant and pandrug-resistant bacteria: An international expert proposal for interim standard definitions for acquired resistance. Clin Microbiol Infect 2012;18:268-281.

22. Andrews JM. Determination of minimum inhibitory concentrations. J Antimicrob Chemother 2001;48(Suppl 1): $5-16$.

23. Labricciosa FM, Sartelli M, Abbo LM, et al. Epidemiology and risk factors for isolation of multi-drug-resistant organisms in patients with complicated intra-abdominal infections. Surg Infect 2018;19:264-272.

24. Buehler SS, Madison B, Snyder SR, et al. Effectiveness of Practices to increase timeliness of providing targeted therapy for inpatients with bloodstream infections: A laboratory medicine best practices systematic review and meta-analysis. Clin Microbiol Rev 2016;29:59-103.

25. De Rosa FG, Corcione S, Pagani N, Di Perri G. From ESKAPE to ESCAPE, From KPC to CCC. Clin Infect Dis 2015;60:1289-290.

26. White BP, Barber KE, Stover KR. Ceftaroline for the treatment of methicillin-resistant Staphylococcus aureus bacteremia. Am J Health Syst Pharm 2017;74:201-208.

27. van Duin D, Bonomo RA. Ceftazidime/avibactam and ceftolozane/tazobactam: Second-generation $\beta$-lactam $/ \beta$ lactamase inhibitor combinations. Clin Infect Dis 2016; 63:234-241.

28. Shields RK, Nguyen MH, Chen L, et al. Ceftazidimeavibactam is superior to other treatment regimens against carbapenem-resistant Klebsiella pneumoniae bacteremia. Antimicrob Agents Chemother 2017;61:e00883-17.

29. Cluck D, Lewis P, Stayer B, et al. Ceftolozanetazobactam: A new-generation cephalosporin. Am J Health Syst Pharm 2015;72:2135-2146.

30. Hackel MA, Lomovskaya O, Dudley MN, et al. In vitro activity of meropenem-vaborbactam against clinical isolates of KPC-positive Enterobacteriaceae. Antimicrob Agents Chemother 2018;62(1).

31. Svoboda P, Kantorová I, Scheer P, et al. Can procalcitonin help us in timing of re-intervention in septic patients after multiple trauma or major surgery? Hepatogastroenterology 2007;54:359-563.

32. Novotny AR, Emmanuel K, Hueser N, et al. Procalcitonin ratio indicates successful surgical treatment of abdominal sepsis. Surgery 2009;145:20-26.

33. Rattan R, Allen CJ, Sawyer RG, et al. Patients with risk factors for complications do not require longer antimi- 
crobial therapy for complicated intra-abdominal infection. Am Surg 2016;82:860-866.

34. Rattan R, Allen CJ, Sawyer RG, et al. Percutaneously drained intra-abdominal infections do not require longer duration of antimicrobial therapy. J Trauma Acute Care Surg 2016;81:108-113.

35. Rattan R, Allen CJ, Sawyer RG, et al. Patients with complicated intra-abdominal infection presenting with sepsis do not require longer duration of antimicrobial therapy. J Am Coll Surg 2016;222:440-446.

36. Zhang T, Wang Y, Yang Q, Dong Y. Procalcitonin-guided antibiotic therapy in critically ill adults: A meta-analysis. BMC Infect Dis 2017; 17:514.

37. Huang H-B, Peng J-M, Weng L, et al. Procalcitoninguided antibiotic therapy in intensive care unit patients: A systematic review and meta-analysis. Ann Intensive Care 2017;7:114.

38. Wirz Y, Meier MA, Bouadma L, et al. Effect of procalcitonin-guided antibiotic treatment on clinical outcomes in intensive care unit patients with infection and sepsis patients: A patient-level meta-analysis of randomized trials. Crit Care Lond Engl 2018;22:191.

39. Schuetz P, Wirz Y, Sager R, et al. Procalcitonin to initiate or discontinue antibiotics in acute respiratory tract infections. Cochrane Database Syst Rev 2017;10:CD007498.

40. Zhuang Y, Peng H, Chen Y, et al. Dynamic monitoring of monocyte HLA-DR expression for the diagnosis, prognosis, and prediction of sepsis. Front Biosci Landmark Ed 2017;22:1344-1354.

41. Rhodes A, Cecconi M. Cell-free DNA and outcome in sepsis. Crit Care 2012;16:170.

42. Blauwkamp TA, Thair S, Rosen MJ, et al. Analytical and clinical validation of a microbial cell-free DNA sequencing test for infectious disease. Nat Microbiol 2019;4: 663-674.

43. May JM, Harrison FE. Role of vitamin C in the function of the vascular endothelium. Antioxid Redox Signal 2013; 19:2068-2083.

44. Patak P, Willenberg HS, Bornstein SR. Vitamin C is an important cofactor for both adrenal cortex and adrenal medulla. Endocr Res 2004;30:871-875.

45. Marik PE. Vitamin C for the treatment of sepsis: The scientific rationale. Pharmacol Ther 2018;189:63-70.

46. Carr AC, Rosengrave PC, Bayer S, et al. Hypovitaminosis $\mathrm{C}$ and vitamin $\mathrm{C}$ deficiency in critically ill patients despite recommended enteral and parenteral intakes. Crit Care Lond Engl 2017;21:300.

47. Fowler AA, Syed AA, Knowlson S, et al. Phase I safety trial of intravenous ascorbic acid in patients with severe sepsis. J Transl Med 2014;12:32.

48. Zabet MH, Mohammadi M, Ramezani M, Khalili H. Effect of high-dose Ascorbic acid on vasopressor's requirement in septic shock. J Res Pharm Pract 2016;5:94-100.

49. Marik PE, Khangoora V, Rivera R, et al. Hydrocortisone, vitamin $\mathrm{C}$, and thiamine for the treatment of severe sepsis and septic shock: A retrospective before-after study. Chest 2017;151:1229-238.

50. Li J. Evidence is stronger than you think: A meta-analysis of vitamin C use in patients with sepsis. Crit Care Lond Engl 2018;22:258.

51. Rhodes A, Evans LE, Alhazzani W, et al. Surviving Sepsis Campaign: International Guidelines for Management of Sepsis and Septic Shock: 2016. Crit Care Med 2017;45: 486-552.
52. Khanna A, English SW, Wang XS, et al. Angiotensin II for the treatment of vasodilatory shock. N Engl J Med 2017;377:419-430.

53. Fernandes D, Sordi R, Pacheco LK, et al. Late, but not early, inhibition of soluble guanylate cyclase decreases mortality in a rat sepsis model. J Pharmacol Exp Ther 2009;328:991-999.

54. Dellinger RP. Inflammation and coagulation: Implications for the septic patient. Clin Infect Dis 2003;36:1259-1265.

55. Symeonides S, Balk RA. Nitric oxide in the pathogenesis of sepsis. Infect Dis Clin North Am 1999;13:449-463.

56. Paciullo CA, McMahon Horner D, Hatton KW, Flynn JD. Methylene blue for the treatment of septic shock. Pharmacotherapy 2010;30:702-715.

57. Alejandria MM, Lansang MAD, Dans LF, Mantaring JB. Intravenous immunoglobulin for treating sepsis, severe sepsis and septic shock. Cochrane Database Syst Rev 2013;(9):CD001090.

58. Liu P, Wu Q, Tang Y, et al. The influence of esmolol on septic shock and sepsis: A meta-analysis of randomized controlled studies. Am J Emerg Med 2018;36:470-474.

59. Monard C, Rimmelé T, Ronco C. Extracorporeal blood purification therapies for sepsis. Blood Purif 2019; 47(Suppl 3):1-14.

60. Hadem J, Hafer C, Schneider AS, et al. Therapeutic plasma exchange as rescue therapy in severe sepsis and septic shock: Retrospective observational single-centre study of 23 patients. BMC Anesthesiol 2014;14:24.

61. Dellinger RP, Carlet JM, Masur H, et al. Surviving Sepsis Campaign guidelines for management of severe sepsis and septic shock. Intensive Care Med. 2004 Apr;30(4): 536-55.

62. Dellinger RP, Levy MM, Carlet JM, et al. Surviving Sepsis Campaign: international guidelines for management of severe sepsis and septic shock: 2008. Intensive Care Med. 2008 Jan;34(1):17-60.

63. Dellinger RP, Levy MM, Rhodes A, et al. Surviving sepsis campaign: international guidelines for management of severe sepsis and septic shock: 2012. Crit Care Med. 2013 Feb;41(2):580-637.

64. Levy MM, Rhodes A, Phillips GS, et al. Surviving Sepsis Campaign: association between performance metrics and outcomes in a 7.5-year study. Crit Care Med 2015;43:3-12.

65. Cecconi M, De Backer D, Antonelli M, et al. Consensus on circulatory shock and hemodynamic monitoring. Task force of the European Society of Intensive Care Medicine. Intensive Care Med 2014;40:1795-815.

66. ProCESS Investigators, Yealy DM, Kellum JA, Huang DT, et al. A randomized trial of protocol-based care for early septic shock. N Engl J Med 2014;370:1683-1693.

67. ARISE Investigators, ANZICS Clinical Trials Group, Peake SL, Delaney A, Bailey M, et al. Goal-directed resuscitation for patients with early septic shock. N Engl J Med 2014;371:1496-1506.

68. Mouncey PR, Osborn TM, Power GS, et al. Trial of early, goal-directed resuscitation for septic shock. N Engl J Med 2015;372:1301-1311.

69. Angus DC, Barnato AE, Bell D, et al. A systematic review and meta-analysis of early goal-directed therapy for septic shock: The ARISE, ProCESS and ProMISe Investigators. Intensive Care Med 2015;41:1549-1560.

70. Monnet X, Marik P, Teboul J-L. Passive leg raising for predicting fluid responsiveness: A systematic review and meta-analysis. Intensive Care Med 2016;42:1935-1947. 
71. Marik PE. Fluid responsiveness and the six guiding principles of fluid resuscitation. Crit Care Med 2016;44: 1920-1922.

72. Monnet X, Teboul J-L. Passive leg raising: five rules, not a drop of fluid! Crit Care Lond Engl 2015;19:18.

73. Cecconi M, De Backer D, Antonelli M, et al. Consensus on circulatory shock and hemodynamic monitoring. Task force of the European Society of Intensive Care Medicine. Intensive Care Med 2014;40:1795-1815.

74. Levy MM, Evans LE, Rhodes A. The Surviving Sepsis Campaign Bundle: 2018 update. Crit Care Med 2018;46: 997-1000.

75. Marik PE, Farkas JD, Spiegel R, et al. POINT: Should the Surviving Sepsis Campaign Guidelines be retired? Yes. Chest 2019;155:12-14.

76. PulmCCM. Hospitals should not implement one-hour sepsis bundles, say SCCM and ACEP. PulmCCM. 2019. https://pulmccm.org/critical-care-review/hospitals-shouldnot-implement-one-hour-sepsis-bundles-say-sccm-andacep/ (Last accessed May 7, 2019).

77. Sign Petition: Surviving Sepsis-Your new treatment guidelines are dangerous! www.thepetitionsite.com/772/ 830/097/surviving-sepsis-campaign-sccm-esicm/ (Last accessed May 7, 2019).

78. Petition to retire the surviving sepsis campaign guidelines. EMCrit Project. 2018. https://emcrit.org/pulmcrit/sscpetition/ (Last accessed May 7, 2019).

79. Martin GS, Mannino DM, Eaton S, Moss M. The epidemiology of sepsis in the United States from 1979 through 2000. N Engl J Med 2003;348:1546-1554.

80. Dombrovskiy VY, Martin AA, Sunderram J, Paz HL. Rapid increase in hospitalization and mortality rates for severe sepsis in the United States: A trend analysis from 1993 to 2003. Crit Care Med 2007;35:1244-1250.

81. Rangel-Frausto MS, Pittet D, Costigan M, et al. The natural history of the systemic inflammatory response syndrome (SIRS). A prospective study. JAMA 1995;273: 117-123.

82. Nesseler N, Defontaine A, Launey Y, et al. Long-term mortality and quality of life after septic shock: A followup observational study. Intensive Care Med 2013;39: 881-888.

83. Wang T, Derhovanessian A, De Cruz S, et al. Subsequent infections in survivors of sepsis: Epidemiology and outcomes. J Intensive Care Med 2014;29:87-95.

84. Prescott HC, Langa KM, Liu V, et al. Increased 1-year healthcare use in survivors of severe sepsis. Am J Respir Crit Care Med 2014;190:62-69.

85. Ou S-M, Chu H, Chao P-W, et al. Long-Term mortality and major adverse cardiovascular events in sepsis survivors. A nationwide population-based study. Am J Respir Crit Care Med 2016;194:209-217.

86. Freund Y, Lemachatti N, Krastinova E, et al. Prognostic accuracy of Sepsis-3 criteria for in-hospital mortality among patients with suspected infection presenting to the emergency department. JAMA 2017;317:301-308.

87. Hwang SY, Jo IJ, Lee SU, et al. Low accuracy of positive qSOFA criteria for predicting 28-day mortality in critically ill septic patients during the early period after emergency department presentation. Ann Emerg Med 2018;71: $1-9$.

88. Churpek MM, Snyder A, Han X, et al. Quick sepsisrelated organ failure assessment, systemic inflammatory response syndrome, and early warning scores for detecting clinical deterioration in infected patients outside the intensive care unit. Am J Respir Crit Care Med 2017;195: 906-911.

89. Raith EP, Udy AA, Bailey M, et al. Prognostic accuracy of the SOFA score, SIRS criteria, and qSOFA score for in-hospital mortality among adults with suspected infection admitted to the intensive care unit. JAMA 2017;317: 290-300.

90. Haydar S, Spanier M, Weems P, et al. Comparison of QSOFA score and SIRS criteria as screening mechanisms for emergency department sepsis. Am J Emerg Med 2017; 35):1730-1733.

91. Sangji NF, Bohnen JD, Ramly EP, et al. Derivation and validation of a novel Emergency Surgery Acuity Score (ESAS). J Trauma Acute Care Surg 2016;81:213-220.

92. Peponis T, Bohnen JD, Sangji NF, et al. Does the emergency surgery score accurately predict outcomes in emergent laparotomies? Surgery 2017;162:445-452.

93. Nandan AR, Bohnen JD, Sangji NF, et al. The Emergency Surgery Score (ESS) accurately predicts the occurrence of postoperative complications in emergency surgery patients. J Trauma Acute Care Surg 2017;83:84-89.

94. Han K, Lee JM, Achanta A, et al. Emergency surgery score accurately predicts the risk of post-operative infection in emergency general surgery. Surg Infect 2019;20: 4-9.

95. Bertsimas D, Dunn J, Velmahos GC, Kaafarani HMA. Surgical risk is not linear: Derivation and validation of a novel, user-friendly, and machine-learning-based Predictive OpTimal Trees in Emergency Surgery Risk (POTTER) calculator. Ann Surg 2018;268:574-583.

96. Gultepe E, Green JP, Nguyen H, et al. From vital signs to clinical outcomes for patients with sepsis: A machine learning basis for a clinical decision support system. J Am Med Inform Assoc 2014;21:315-325.

97. Horng S, Sontag DA, Halpern Y, et al. Creating an automated trigger for sepsis clinical decision support at emergency department triage using machine learning. PloS One 2017; 12:e0174708.

98. Nemati S, Holder A, Razmi F, et al. An interpretable machine learning model for accurate prediction of sepsis in the ICU. Crit Care Med 2018;46:547-553.

99. Mao Q, Jay M, Hoffman JL, et al. Multicentre validation of a sepsis prediction algorithm using only vital sign data in the emergency department, general ward and ICU. BMJ Open 2018;8:e017833.

100. Chiew CJ, Liu N, Tagami T, et al. Heart rate variability based machine learning models for risk prediction of suspected sepsis patients in the emergency department. Medicine (Baltimore) 2019;98:e14197.

101. Barton C, Chettipally U, Zhou Y, et al. Evaluation of a machine learning algorithm for up to 48-hour advance prediction of sepsis using six vital signs. Comput Biol Med 2019;109:79-84.

Address correspondence to: Dr. Tanya L. Zakrison Section for Trauma and Acute Care Surgery The University of Chicago Medicine 5841 South Maryland Avenue Chicago, IL 60637

E-mail: tzakrison@surgery.bsd.uchicago.edu 